\title{
Galactomannan in Bronchoalveolar Lavage Fluid for Diagnosis of Invasive Pulmonary Aspergillosis with Nonneutropenic Patients
}

\author{
Qidong Zhuang, ${ }^{1}$ Hongying Ma, ${ }^{1}$ Yun Zhang, ${ }^{1}$ Lei Chen, ${ }^{1}$ Li Wang, ${ }^{2}$ Lin Zheng, \\ Zaichun Deng, ${ }^{1}$ and Zhongbo Chen ${ }^{1}$ \\ ${ }^{1}$ Department of Pulmonology, The Affiliated Hospital of Ningbo University, 247 Renmin Road, Ningbo, Zhejiang 315020, China \\ ${ }^{2}$ Department of Microbiology, The Affiliated Hospital of Ningbo University, 247 Renmin Road, Ningbo, Zhejiang 315020, China
}

Correspondence should be addressed to Zaichun Deng; leonczb@sina.com and Zhongbo Chen; leonczb@yahoo.com

Received 8 July 2017; Revised 4 September 2017; Accepted 12 September 2017; Published 13 November 2017

Academic Editor: Alice M. Turner

Copyright (c) 2017 Qidong Zhuang et al. This is an open access article distributed under the Creative Commons Attribution License, which permits unrestricted use, distribution, and reproduction in any medium, provided the original work is properly cited.

Background. We evaluated the utility of galactomannan (GM) in bronchoalveolar lavage fluid (BALF) for the diagnosis of invasive pulmonary aspergillosis (IPA) in nonneutropenic patients. Methods. A total of 183 patients were included in the final analysis. Bronchoscopies and the detection of GM in BALF were all performed on them. Results. Ten cases of IPA were diagnosed. ROC data demonstrated that, for diagnosing IPA, an optimal cutoff value for GM in BALF of 0.76 yielded a sensitivity of $100.0 \%$ and a specificity of $76.2 \%$. Symptoms and radiological findings had no significant difference between proven or probable IPA group and non-IPA group. In our case-control analysis, although nine patients with false-positive results received treatment with Piperacillin/tazobactam, there was no significant difference between case and control group. Conclusions. BALF GM detection is a valuable adjunctive diagnostic tool. Our retrospective study suggests that the optimal value of GM detection in BALF is 0.76 in nonneutropenic patients.

\section{Introduction}

Aspergillus is a ubiquitous fungus which can cause various kinds of pulmonary aspergillosis, such as allergic bronchopulmonary aspergillosis (ABPA), pulmonary aspergilloma (PA), and invasive pulmonary aspergillosis [1] (IPA). IPA is the most severe type in the pulmonary aspergillosis. Moreover, it is an important cause of fatality in immunocompromised populations, especially in hematological and solidorgan transplant patients [2]. Once a study reported that the morbidity of IPA might be $15 \%$ to $20 \%$ in HSCT recipients and neutropenic patients with hematologic malignancies, and the mortality can be as high as $50 \%$ to $90 \%$ [3]. In the past, neutropenia was considered a major risk for IPA. However, recent studies reported neutropenia was absent in a number of IPA patients $[4,5]$. In nonneutropenic patients, chronic obstructive pulmonary disease (COPD) was the most common underlying disease [4]. Besides, 19.2\%-22.2\% patients with IPA had no diseases, whose fatality rate was as high as $33.3 \%$ to $39 \%$. Current evidence suggests that antifungal therapy should be initiated as early as possible to improve outcome. So early detection, timely diagnosis, and early treatment are the keys to decrease the mortality.

As the development of chest computer tomography, a variety of pulmonary diseases can be diagnosed. The classic halo sign and air crescent sign are frequent in neutropenic hosts with IPA. However, the above signs are rare and less specific among nonneutropenic patients [6]. Moreover, pulmonary consolidation, infiltration, and nodular lesions are common. The current gold standard for the diagnosis of proven IPA requires histological evidence. But the invasive procedures carry high risks for the patients with underlying diseases. The microbiological culture is time-consuming and insensitive [7-9]. So it is urgent for us to seek a new method to detect the Aspergillus.

Galactomannan (GM) is a polysaccharide component of the cell wall of Aspergillus [7], which can be released into the body fluids by growing hyphae, such as blood, urine, and 
bronchoalveolar fluid (BALF). With using in vitro and animal models of IPA, the researchers suggested that GM might be released at an earlier stage and at a higher concentration into the BALF than in serum [8]. Currently, serum GM assay has been used for supervising, early detection, and timely diagnosis [10] in neutropenic hosts. However, the diagnostic value of BALF GM assay for IPA is still uncertain, especially in the nonneutropenic patients. To further investigate the utility of GM in BALF for the diagnosis of IPA in nonneutropenic hosts, we conducted this retrospective study.

\section{Methods}

2.1. Study Population. All nonneutropenic patients coming from Affiliated Hospital of Ningbo University, who were admitted to Respiratory Medicine Department from April 2014 to February 2017, were reviewed. Written informed consent was obtained from each patient. And the Institutional Review Board approved this work.

2.2. Bronchoscopy and Sample Collecting. Experienced doctors performed all bronchoscopies with bronchoalveolar lavage ( $20 \mathrm{ml}$ for three times). The site of bronchoscopy was guided by the lung CT scan at first. Based on the infiltration location on the chest radiograph, the sampling area was selected. If there were diffuse lesions in bilateral lungs, the bronchoscope would be wedged into the middle lobe. If there were multiple lesions in bilateral lungs, the bronchoscope would be wedged into the most serious segment of left and right lung, respectively; then the lavage samples were mixed. If there were multiple lesions in unilateral lung, the bronchoscope would be wedged into the most serious segment or the involved segments and then the lavage samples mixed.

The lavage samples were submitted for direct microscopic examination and microbiological culture. Besides, a vortex was done for the remaining BALF samples and the supernatant was used for GM detection by using the Platelia Aspergillus EIA (Bio-Rad). An OD index $\geqq 0.8$ for BALF GM was considered positive $[2,11,12]$.

2.3. Case Definitions. Each of the included IPA patients was classified based on the criteria of the European Organization for Research and Treatment of Cancer and the Mycoses Study Group (EORTC/MSG) revised in 2008. Proven cases required histopathological evidence. Probable cases required at least a host factor, a radiological criterion, and a kind of microbiological evidence. Possible cases only required hosts and radiological criteria without microbiological proofs.

Receipt of an allogeneic stem cell transplantation, prolonged use of corticosteroids, hematological diseases, solidorgan transplantation, and treatment with $\mathrm{T}$ cell immunosuppressants were classic risk factors. In recent years, critically ill patients with COPD [2] and patients with cirrhosis [13] were also host factors. Moreover, diabetes mellitus, rheumatologic disease, and bronchiectasis had been reported as the underlying diseases for IPA [4]. Patients with above diseases were considered as host factors, except for neutropenic patients. Because the imaging manifestations were less specific for nonneutropenic groups with IPA, pulmonary consolidation, infiltration, or nodular lesions were considered the radiological criteria. Based on previous studies [14-16], the modified microbiological criteria included any of the following: (1) positive isolation of Aspergillus species from sputum or BALF by culture; (2) a positive serum GM result (ODI $\geqq 0.5)$ verified in two consecutive samples; (3) a positive BALF GM result (ODI $\geqq 0.8$ ) with, simultaneously, antibiotic treatment invalid but antifungal treatment against aspergillosis valid.

The "non-IPA" patients included those with ABPA, aspergilloma, and the colonization or contamination, except for the IPA patients. To compare the characteristics of IPA patients with that of non-IPA ones, patients in whom there was no evidence of IPA were classified as the "nonIPA" group, which excluded ABPA, aspergilloma, and the colonization or contamination group. Colonization or contamination group was defined as positive Aspergillus culture from sputum or BALF, who clinically improved without antifungal therapy.

2.4. Case-Control Analysis. To analyze the influences of different risk factors on BALF GM detection, non-IPA patients were divided into two groups: patients with positive BALF GM results (case patients) and patients with negative BALF GM results (control patients). Both groups excluded the IPA, ABPA, aspergilloma, and the colonization or contamination patients.

2.5. Statistical Analysis. BALF GM assay should be calculated sensitivity, specificity, positive predictive index (PPV), and negative predictive index (NPV). Although we considered an ODI ratio $\geqq 0.8$ in BALF GM as the positive result for referenced diagnosis of IPA, the receiver operating characteristic (ROC) curve was used to acquire the optimal cutoff index. Furthermore, factors causing false-positive results in the BALF GM assay were analyzed. Quantitative variables were compared using $t$-tests. For qualitative date, Pearson's $\chi^{2}$ or Fisher's exact tests were used. Logistic regression analysis was performed to identify risk factors for false-positive BALF GM results. All tests used were two-tailed, and a $p$ value of $<0.05$ was considered statistically significant.

\section{Results}

3.1. Patient Characteristics. During the study period, a total of 226 inpatients were enrolled in. Of those, 43 patients were excluded from the study, including 30 patients without the performance of bronchoscopy, 1 patient with lack clinical information, and 12 patients without undergoing the BALF GM assay. Thus, 183 patients participated in our final analysis (Figure 1). The baseline clinical characteristics of chosen patients with IPA and "non-IPA" groups are shown in Table 1. IPA patients were classified as proven IPA $(n=0)$, probable IPA $(n=10)$, and possible IPA $(n=21)$. Non-IPA patients included non-IPA $(n=143)$, ABPA $(n=1)$, aspergilloma $(n=3)$, and colonization or contamination hosts $(n=5)$.

In the proven, probable, and possible IPA patients, the pulmonary diseases were the main underlying condition, such as emphysema $(n=5)$, COPD $(n=1)$, pulmonary 
TABLE 1: Baseline clinical characteristics of the study population ${ }^{\mathrm{a}}$.

\begin{tabular}{|c|c|c|}
\hline & $\begin{array}{c}\text { IPA } \\
(n=31), n(\%)\end{array}$ & $\begin{array}{c}\text { Non-IPA } \\
(n=152), n \\
(\%)\end{array}$ \\
\hline Age, mean years & 58.97 & 55.62 \\
\hline Male gender & $20(64.5)$ & $82(53.9)$ \\
\hline High-risk environment exposed & $2(6.5)$ & $1(0.7)$ \\
\hline \multicolumn{3}{|l|}{ Underlying disease } \\
\hline Emphysema & $5(16.1)$ & $22(14.5)$ \\
\hline COPD & $1(3.2)$ & $8(5.3)$ \\
\hline Bronchial asthma & 0 & $8(5.3)$ \\
\hline Pulmonary tuberculosis & $2(6.5)$ & $10(6.6)$ \\
\hline Solid tumor & $3(9.7)$ & $8(5.3)$ \\
\hline Bronchiectasis & $3(9.7)$ & $24(15.8)$ \\
\hline Diabetes & $5(16.1)$ & $16(10.5)$ \\
\hline Liver cirrhosis & 0 & $1(0.7)$ \\
\hline Hematologic malignancy & 0 & $1(0.7)$ \\
\hline Autoimmune disease & $2(6.5)$ & $2(1.3)$ \\
\hline Kidney non-malignant disease & 0 & $3(2.0)$ \\
\hline Organ failure & $2(6.5)$ & $1(0.7)$ \\
\hline No underlying disease & $9(29.0)$ & $57(37.5)$ \\
\hline \multicolumn{3}{|l|}{ Final diagnosis } \\
\hline & Proven: 0 & ABPA: $1(0.7)$ \\
\hline & $\begin{array}{c}\text { Probable: } 10 \\
(32.3)\end{array}$ & $\begin{array}{c}\text { Aspergilloma: } \\
3(2.0)\end{array}$ \\
\hline & $\begin{array}{l}\text { Possible: } 21 \\
\quad(67.7)\end{array}$ & $\begin{array}{c}\text { Colonization } \\
\text { or contami- } \\
\text { nation: } 5 \\
(3.3)\end{array}$ \\
\hline & & $\begin{array}{c}\text { Others: } 143 \\
\quad(94.1)\end{array}$ \\
\hline
\end{tabular}

IPA, invasive pulmonary aspergillosis; COPD, chronic obstructive pulmonary disease; ABPA, allergic bronchopulmonary aspergillosis. ${ }^{\mathrm{a}}$ Date are number (\%) of patients, unless otherwise indicated.

tuberculosis $(n=2)$, bronchiectasis $(n=3)$, and lung cancer $(n=3)$. Nine (29.0\%) patients had no underlying diseases.

3.2. Clinical Symptoms and Radiological Findings. Table 2 shows the prominent symptoms and radiological findings in patients with proven or probable IPA. Each patient complaint about at least one of following symptoms: cough $(n=9$, $90.0 \%)$, dyspnoea $(n=3,30.0 \%)$, fever $(n=2,20.0 \%)$, haemoptysis $(n=2,20.0 \%)$, chest tightness $(n=1$, $10.0 \%)$, and chest pain $(n=1,10.0 \%)$. Chest computed tomography (CT) was performed in all included patients. The most frequent abnormality was infiltrate or nodules ( $n=8,80.0 \%)$. Common imaging findings occurring in neutropenic patients, such as halo sign and air crescent sign, were observed in only one patient. However, there was no significant difference between proven or probable IPA group and non-IPA group. Besides, there was also no significant difference in seasonal distribution.
TABLE 2: Comparison of seasonal distribution, clinical symptoms, and chest CT between proven/probable IPA patients and non-IPA patients.

\begin{tabular}{lccc}
\hline Characteristics & $\begin{array}{c}\text { Proven/probable } \\
\text { IPA } \\
(n=10)\end{array}$ & $\begin{array}{c}\text { Non-IPA } \\
(n=143)\end{array}$ & $p$ \\
\hline Age $\geq 60$ years & $6(60.0)$ & $63(44.1)$ & 0.515 \\
Male gender & $8(80.0)$ & $77(53.8)$ & 0.201 \\
Seasonal distribution, $n$ (\%) & & & \\
$\quad$ March-October & $7(70.0)$ & $93(65.0)$ & 1.000 \\
Clinical symptoms, $n(\%)$ & & & \\
Cough & $9(90.0)$ & $101(70.6)$ & 0.340 \\
Fever & $2(20.0)$ & $37(25.9)$ & 0.971 \\
Dyspnoea & $3(30.0)$ & $16(11.2)$ & 0.212 \\
Haemoptysis & $2(20.0)$ & $21(14.7)$ & 1.000 \\
Chest tightness & $1(10.0)$ & $12(8.4)$ & 0.600 \\
Chest pain & $1(10.0)$ & $18(12.6)$ & 1.000 \\
Chest CT, $n$ (\%) & & & \\
Infiltrate/Nodules & $8(80.0)$ & $100(69.9)$ & 0.751 \\
Bronchiectasis & $3(30.0)$ & $28(19.6)$ & 0.700 \\
Cavity & $3(30.0)$ & $18(12.6)$ & 0.284 \\
Air crescent sign & $1(10.0)$ & 0 & 0.065 \\
Halo sign & $1(10.0)$ & $10(7.0)$ & 0.537 \\
Other & $1(10.0)$ & $12(8.4)$ & 0.600 \\
\hline
\end{tabular}

IPA, invasive pulmonary aspergillosis; CT, computer tomography. ${ }^{a}$ NonIPA patients are defined as individuals in the "non-IPA" group excluding aspergilloma, ABPA, colonization, or contamination patients.

TABLE 3: Performance of GM detection for diagnosis of IPA in BALF.

\begin{tabular}{lcccc}
\hline Cutoff value & Sensitivity\% & Specificity\% & PPV\% & NPV\% \\
\hline BALF GM $\geq 0.5$ & $100.0 \%$ & $64.3 \%$ & $16.4 \%$ & $100.0 \%$ \\
BALF GM $\geq 0.76$ & $100.0 \%$ & $76.2 \%$ & $22.7 \%$ & $100.0 \%$ \\
BALF GM $\geq 0.8$ & $90.0 \%$ & $78.3 \%$ & $22.5 \%$ & $99.1 \%$ \\
BALF GM $\geq 1.0$ & $70.0 \%$ & $82.5 \%$ & $21.9 \%$ & $97.5 \%$ \\
BALF GM $\geq 1.5$ & $60.0 \%$ & $86.0 \%$ & $23.1 \%$ & $96.8 \%$ \\
BALF GM $\geq 2.0$ & $50.0 \%$ & $88.8 \%$ & $23.8 \%$ & $96.2 \%$ \\
\hline
\end{tabular}

GM, galactomannan; IPA, invasive pulmonary aspergillosis; BALF, bronchoalveolar lavage fluid; PPV, positive predictive value; NPV, negative predictive value.

3.3. Performance of BALF GM Assay. The sensitivity, specificity, PPV, and NPV of BALF GM assay are shown in Table 3. We considered proven and probable IPA as true-positives. When the OD index cutoff to define BALF GM positivity was lowered from $\geq 2.0$ to $\geq 0.5$, the estimated sensitivity increased from $50.0 \%$ to $100.0 \%$, and the specificity decreased from $88.8 \%$ to $64.3 \%$. At the index cutoff value of $\geq 0.8$, the BALF GM assay had a sensitivity of $90 \%$ and a specificity of $78.3 \%$. ROC data demonstrated that, for diagnosing IPA, an optimal cutoff value for GM in BALF of 0.76 yielded a sensitivity of $100.0 \%$ and a specificity of $76.2 \%$, and that the diagnostic accuracy of BALF GM as determined by the area under the ROC curve was 0.88 (95\% CI 0.82-0.94) (Figure 2). 
TABLE 4: Risk factors for false-positive galactomannan results in bronchoalveolar lavage assays with univariate analysis and logistic regression analysis, respectively.

\begin{tabular}{|c|c|c|c|c|}
\hline \multirow{2}{*}{ Variables } & \multirow{2}{*}{$\begin{array}{l}\text { Case patients }^{\mathrm{a}} \\
(n=30), n(\%)\end{array}$} & \multirow{2}{*}{$\begin{array}{l}\text { Control patients }^{\mathrm{b}} \\
(n=113), n(\%)\end{array}$} & \multicolumn{2}{|c|}{$p$} \\
\hline & & & Univariate analysis & Logistic regression analysis \\
\hline Age $\geq 60$ years & $12(40.0)$ & $51(45.1)$ & 0.615 & 0.533 \\
\hline Male gender & $13(43.3)$ & $64(56.6)$ & 0.194 & 0.067 \\
\hline \multicolumn{5}{|l|}{ Seasonal distribution } \\
\hline March-October & $23(76.7)$ & $70(61.9)$ & 0.133 & 0.129 \\
\hline \multicolumn{5}{|l|}{ Underlying disease } \\
\hline Emphysema & $2(6.7)$ & $10(8.8)$ & 0.990 & 0.608 \\
\hline COPD & $3(10.0)$ & $5(4.4)$ & 0.463 & 0.121 \\
\hline Bronchial asthma & $2(6.7)$ & $5(4.4)$ & 0.976 & 0.273 \\
\hline Pulmonary tuberculosis & $7(23.3)$ & $10(8.8)$ & 0.063 & 0.117 \\
\hline Solid tumor & 0 & $11(9.7)$ & 0.164 & 0.999 \\
\hline Bronchiectasis & $7(23.3)$ & $16(14.2)$ & 0.349 & 0.431 \\
\hline Diabetes & 0 & $11(9.7)$ & 0.164 & 0.999 \\
\hline Liver cirrhosis & 0 & $1(0.9)$ & 1.000 & 0.999 \\
\hline Hematologic malignancy & 0 & $1(0.9)$ & 1.000 & 1.000 \\
\hline Autoimmune disease & 0 & $2(1.8)$ & 1.000 & 0.999 \\
\hline Kidney non-malignant disease & $1(3.3)$ & $1(0.9)$ & 0.377 & 1.000 \\
\hline \multicolumn{5}{|l|}{ Antibiotics } \\
\hline Piperacillin/tazobactam & $9(30.0)$ & $22(19.5)$ & 0.213 & 0.479 \\
\hline Mezlocillin/sulbactam & $2(6.7)$ & $5(4.4)$ & 0.976 & 0.726 \\
\hline Cephalosporins & $7(23.3)$ & $49(43.4)$ & 0.046 & 0.157 \\
\hline Quinolones & $7(23.3)$ & $37(32.7)$ & 0.321 & 0.404 \\
\hline
\end{tabular}

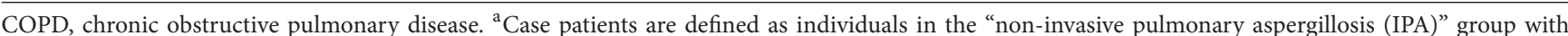
positive bronchoalveolar lavage fluid (BALF) galactomannan (GM) results, excluding aspergilloma, ABPA, colonization, or contamination. Namely, the group is the other diagnosis with positive BALF GM results. ${ }^{b}$ Control patients are defined as individuals in the "non-IPA" group with negative BALF GM results, excluding aspergilloma, colonization, or contamination. Namely, the group is the other diagnosis with negative BALF GM results.

3.4. Case-Control Analysis. The positive BALF GM assay result occurred in 55 patients, 9 were diagnosed as probable IPA, 10 were classified as possible IPA, 1 was considered as ABPA, 2 were diagnosed as aspergilloma, 3 were defined as colonization or contamination, and the remaining 30 were regarded as other diagnoses. To assess the factors of BALF GM assay with false-positive results, we performed a casecontrol analysis. Univariate and multivariate analysis of the date are shown in Table 4. Nine patients with false-positive results were treated with Piperacillin/tazobactam, but there was no significant difference between the case and control group.

\section{Discussion}

IPA is a life-threatening disease, especially in immunocompromised hosts. The classic risk factors for IPA include neutropenia, receipt of an allogeneic stem cell transplant, long-time use of corticosteroids and treatment with immunosuppressants [10]. With the in-depth studies, hematologic diseases [9, 17], COPD [18], liver cirrhosis [19], and intensive care unit patients [20] are also susceptible individuals.
Furthermore, in recent researches $[4,5]$, organ failure, pulmonary tuberculosis, bronchiectasis, and diabetes patients and even those with no underlying diseases can be infected by Aspergillus, which is similar to our study. Notably, in our small sample, $29.0 \%$ of 31 probable/possible IPA patients were void of underlying diseases. Besides, the pulmonary diseases were the most common underlying condition. In patients with airways or lung parenchyma damaged by underlying respiratory diseases, the defense function is limited. When they inhale the Aspergillus into the respiratory tract, the Aspergillus may colonize and even cause IPA. To find out the specific clinical symptoms and radiological presentation, we compared proven/probable IPA patients with non-IPA individuals. Consequently, as previous studies recorded [6, 9], the classic halo sign and air crescent sign were far less common and specific in nonneutropenic ones. Furthermore, there were no significant differences in frequency of various symptoms or imaging findings.

Rapid initiation of systemic antifungal treatment is the key to improve the prognosis of IPA patients. Diagnosis of IPA is always an arduous task, although a large number of reports have demonstrated that the detection of GM in blood samples coming from neutropenic patients is helpful for early 


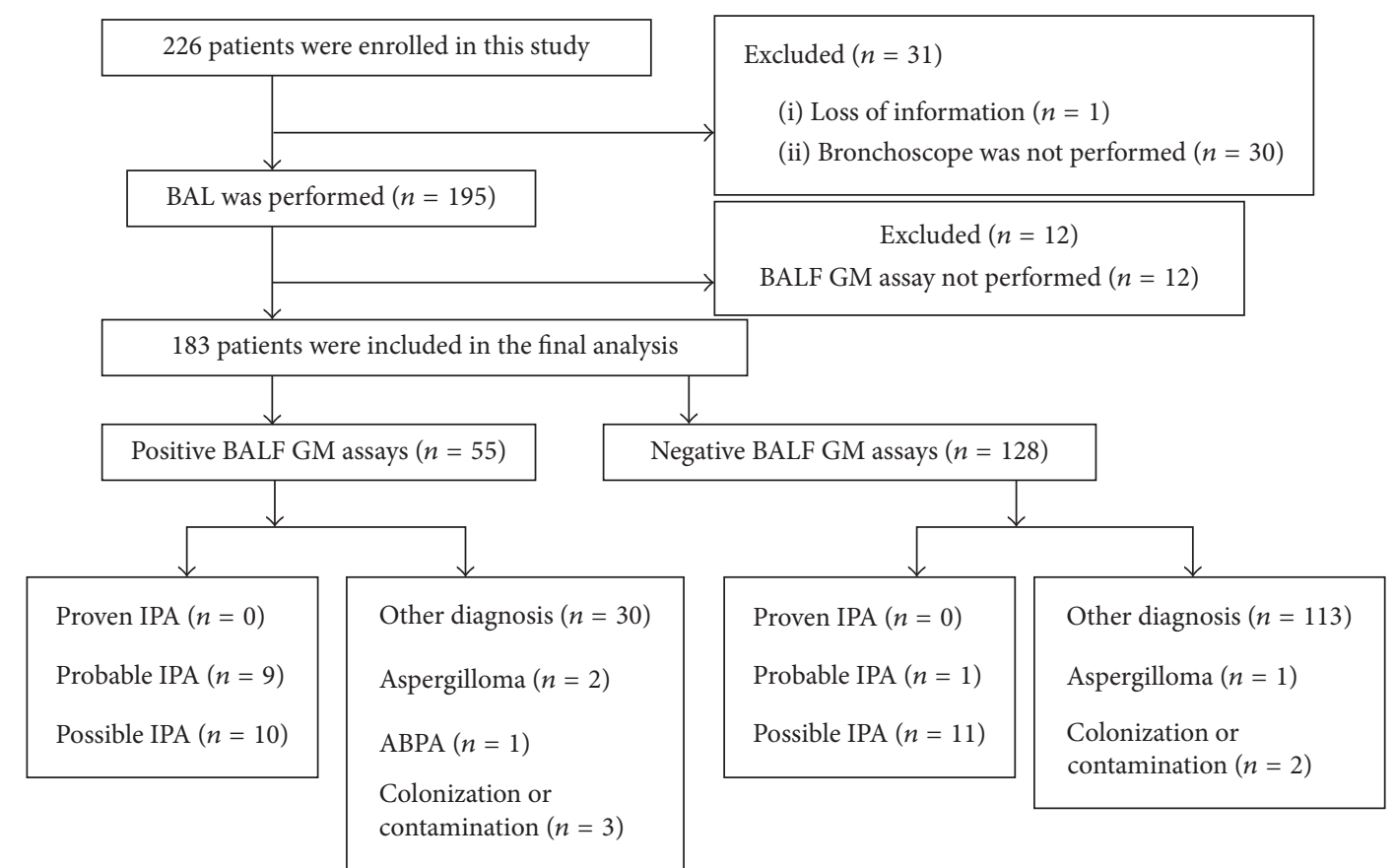

FIGURE 1: Study flow diagram. BAL, bronchoalveolar lavage; BALF, bronchoalveolar lavage fluid; GM, galactomannan; IPA, invasive pulmonary aspergillosis; ABPA, allergic bronchopulmonary aspergillosis.

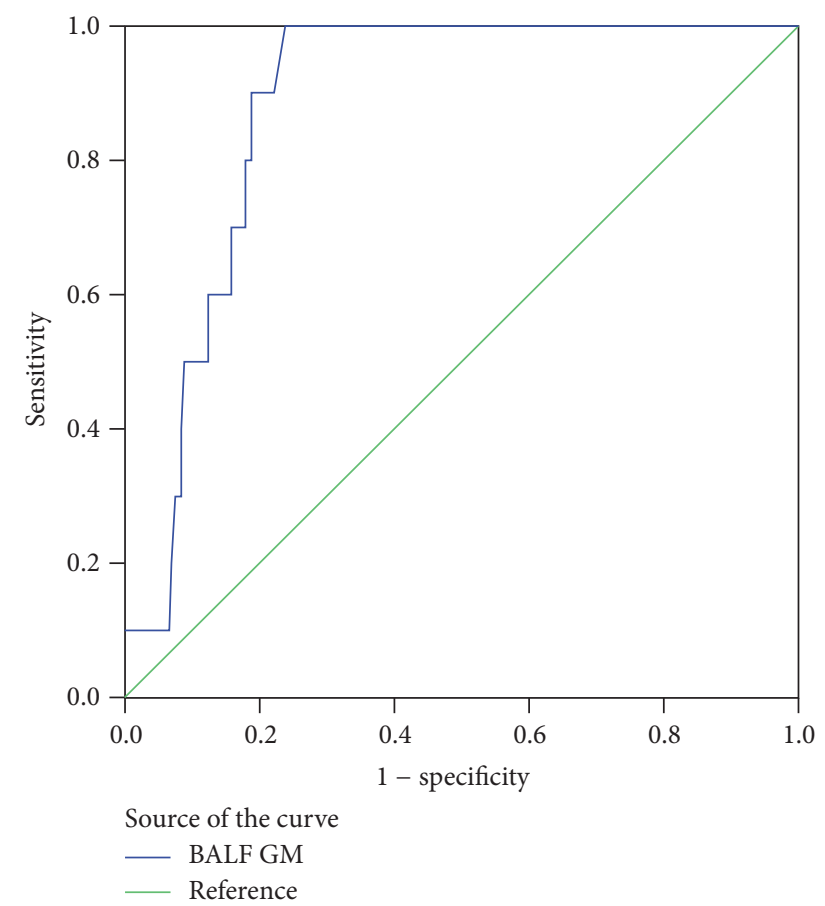

FIGURE 2: Receiver operating characteristic (ROC) curves for galactomannan assay in 183 study populations. Areas under the ROC curve was 0.88 (95\% CI 0.82-0.94).

diagnosis. However, the utility of BALF GM assay still needs to be investigated by many clinical studies, especially in nonneutropenic hosts. Based on the previous findings [2, 3, 7-9, $17,18]$, the optimal cutoff value of BALF GM assay fluctuated, ranging from 0.5 to 1.25 in patients with different underlying diseases. A meta-analysis [19] of detecting galactomannan in BALF suggested the optimal cutoff value was 1.0. In our study, we evaluated the efficacy of measuring GM concentration in BALF for diagnosing IPA in nonneutropenic patients. According to the ROC data, the optimal cutoff index was 0.76 , and the sensitivity and specificity were $100.0 \%$ and $76.2 \%$ for diagnosing IPA, respectively. This cutoff value was consistent with the result of other researches [2,12]. Besides, as previous studies reported, the GM detection of BALF samples had high negative predictive value. In our finding, although the cutoff value ranged from 0.5 to 2.0 , the negative predictive value was always over $96.2 \%$.

Various factors can cause false-positive result in BALF GM detection, such as BALF sample pretreatment with Sputasol [21], antifungal treatment, and $\beta$-lactam antibiotics. $\beta$-Lactam antibiotics, especially Piperacillin/tazobactam and Mezlocillin/sulbactam, were usually used in patients with bacterial pneumonia. As they are semisynthetic drugs derived from natural compounds produced by other molds, many false-positive BALF GM results from patients taking them have been reported [22]. However, in other studies [14, 18], BALF GM false-positive rates were not significantly different between patients treated with $\beta$-lactam antibiotics and those who did not receive these drugs. In our study, although 11 non-IPA patients treated with Piperacillin/tazobactam and Mezlocillin/sulbactam had false-positive results in BALF GM detection, there was no significant difference between the case and control group. Colonization or contamination of the Aspergillus in the respiratory tract may be another reason for false-positive results. We should note that 3 patients 
considered as colonization or contamination had falsepositive values in BALF GM assay. In spite of negative culture results, the patients with underlying pulmonary diseases may be colonized by Aspergillus, which could be another contributing factor for false positivity.

This study has several limitations. First, the present study was retrospective. We may perform a prospective research in the future. Secondly, the number of patients with proven IPA was small; in fact, none of them had proven IPA. Besides, the patients came from a single center. So we will enlarge the number of target population and invite other hospitals to participate in the following research. Thirdly, we used GM detection in BALF as part of mycological criterion for diagnosing, which may cause incorporation bias. If the results of BALF GM were excluded from mycological criteria of IPA, 3 of 10 probable IPA patients were reclassified as possible cases. The sensitivity of BALF GM assay may be higher than it really is.

In conclusion, our retrospective study suggests that the optimal value of GM detection in BALF is 0.76 in nonneutropenic patients. However, the utility of GM in BALF for diagnosing IPA in nonneutropenic hosts still needs to be evaluated by lots of multicenter studies.

\section{Conflicts of Interest}

The authors declare that they have no conflicts of interest.

\section{Authors' Contributions}

Zhongbo Chen and Zaichun Deng participated in study design; Qidong Zhuang, Hongying Ma, Yun Zhang, Lei Chen, Li Wang, and Lin Zheng participated in data collection and analysis; Qidong Zhuang also participated in writing.

\section{Acknowledgments}

This work was supported by Ningbo Social and Scientific Development Fund (no. 2012C50006, no. 2015C50012) and Natural Science Foundation of Ningbo (no. 2016A610132, no. 2017A610250).

\section{References}

[1] S. Y. Park, S. O. Lee, S. H. Choi et al., "Serum and bronchoalveolar lavage fluid galactomannan assays in patients with pulmonary aspergilloma," Clinical Infectious Diseases, vol. 52, no. 7, pp. e149-e152, 2011.

[2] H. He, L. Ding, B. Sun, F. Li, and Q. Zhan, "Role of galactomannan determinations in bronchoalveolar lavage fluid samples from critically ill patients with chronic obstructive pulmonary disease for the diagnosis of invasive pulmonary aspergillosis: a prospective study," Critical Care, vol. 16, no. 4, article R138, 2012.

[3] M. H. Nguyen, H. Leather, C. J. Clancy et al., "Galactomannan testing in bronchoalveolar lavage fluid facilitates the diagnosis of invasive pulmonary aspergillosis in patients with hematologic malignancies and stem cell transplant Recipients," Biology of Blood and Marrow Transplantation, vol. 17, no. 7, pp. 10431050, 2011.
[4] X. Cai, W. Ni, C. Wei, and J. Cui, "Diagnostic value of the serum galactomannan and $(1,3)-\beta$-D-glucan assays for invasive pulmonary aspergillosis in non-neutropenic patients," Internal Medicine, vol. 53, no. 21, pp. 2433-2437, 2014.

[5] Z. Dai, H. Zhao, S. Cai, Y. Lv, and W. Tong, "Invasive pulmonary aspergillosis in non-neutropenic patients with and without underlying disease: a single-centre retrospective analysis of 52 subjects," Respirology, vol. 18, no. 2, pp. 323-331, 2013.

[6] R. Kojima, U. Tateishi, M. Kami et al., "Chest computed tomography of late invasive aspergillosis after allogeneic hematopoietic stem cell transplantation," Biology of Blood and Marrow Transplantation, vol. 11, no. 7, pp. 506-511, 2005.

[7] M. Hoenigl, J. Prattes, B. Spiess et al., "Performance of galactomannan, beta-d-glucan, aspergillus lateral-flow device, conventional culture, and pcr tests with bronchoalveolar lavage fluid for diagnosis of invasive pulmonary aspergillosis," Journal of Clinical Microbiology, vol. 52, no. 6, pp. 2039-2045, 2014.

[8] Z. Racil, I. Kocmanova, M. Toskova et al., "Galactomannan detection in bronchoalveolar lavage fluid for the diagnosis of invasive aspergillosis in patients with hematological diseasesthe role of factors affecting assay performance," International Journal of Infectious Diseases, vol. 15, no. 12, pp. e874-e881, 2011.

[9] J. Maertens, V. Maertens, K. Theunissen et al., "Bronchoalveolar lavage fluid galactomannan for the diagnosis of invasive pulmonary aspergillosis in patients with hematologic diseases," Clinical Infectious Diseases, vol. 49, no. 11, pp. 1688-1693, 2009.

[10] B. De Pauw, T. J. Walsh, and J. P. Donnelly, "Revised definitions of invasive fungal disease from the European organization for research and treatment of cancer/invasive fungal infections cooperative group and the national institute of allergy and infectious diseases mycoses study group (EORTC/MSG) consensus group," Clinical Infectious Diseases, vol. 46, no. 12, pp. 1813-1821, 2008.

[11] S.-C. Heng, S. C.-A. Chen, C. O. Morrissey et al., "Clinical utility of Aspergillus galactomannan and PCR in bronchoalveolar lavage fluid for the diagnosis of invasive pulmonary aspergillosis in patients with haematological malignancies," DIAGNOSTIC MICROBIOLOGY AND INFECTIOUS DISEASE, vol. 79, no. 3, pp. 322-327, 2014.

[12] J. D'Haese, K. Theunissen, E. Vermeulen et al., "Detection of galactomannan in bronchoalveolar lavage fluid samples of patients at risk for invasive pulmonary aspergillosis: Analytical and clinical validity," Journal of Clinical Microbiology, vol. 50, no. 4, pp. 1258-1263, 2012.

[13] A. Russo, S. Giuliano, A. Vena et al., "Predictors of mortality in non-neutropenic patients with invasive pulmonary aspergillosis: Does galactomannan have a role?" DIAGNOSTIC MICROBIOLOGY AND INFECTIOUS DISEASE, vol. 80, no. 1 , pp. 83-86, 2014.

[14] S. Zhang, S. Wang, Z. Wan, R. Li, and J. Yu, "The diagnosis of invasive and noninvasive pulmonary aspergillosis by serum and bronchoalveolar lavage fluid galactomannan assay," BioMed Research International, vol. 2015, Article ID 943691, 2015.

[15] J. Fortún, P. Martín-Dávila, E. Gomez Garcia de la Pedrosa et al., "Galactomannan in bronchoalveolar lavage fluid for diagnosis of invasive aspergillosis in non-hematological patients," Infection, vol. 72, no. 6, pp. 738-744, 2016.

[16] J. Prattes, H. Flick, F. Prüller et al., "Novel tests for diagnosis of invasive aspergillosis in patients with underlying respiratory diseases," American Journal of Respiratory and Critical Care Medicine, vol. 190, no. 8, pp. 922-929, 2014. 
[17] L.-Y. Hsu, Y. Ding, J. Phua et al., "Galactomannan testing of bronchoalveolar lavage fluid is useful for diagnosis of invasive pulmonary aspergillosis in hematology patients.," BMC Infectious Diseases, vol. 10, p. 44, 2010.

[18] X.-B. Zhang, G.-P. Chen, Q.-C. Lin, X. Lin, H.-Y. Zhang, and J.-H. Wang, "Bronchoalveolar lavage fluid galactomannan detection for diagnosis of invasive pulmonary aspergillosis in chronic obstructive pulmonary disease," Medical Mycology, vol. 51, no. 7, pp. 688-695, 2013.

[19] M. Zou, L. Tang, and S. Zhao, "Systematic review and metaanalysis of detecting Galactomannan in bronchoalveolar lavage fluid for diagnosing invasive Aspergillosis," PLoS ONE, vol. 7, no. 8, Article ID e43347, 2012.

[20] W. Meersseman, K. Lagrou, J. Maertens et al., "Galactomannan in bronchoalveolar lavage fluid: a tool for diagnosing aspergillosis in intensive care unit patients," American Journal of Respiratory and Critical Care Medicine, vol. 177, no. 1, pp. 2734, 2008.

[21] J. Prattes, C. Koidl, S. Eigl, R. Krause, and M. Hoenigl, "Bronchoalveolar lavage fluid sample pretreatment with Sputasol ${ }^{5}$ significantly reduces galactomannan levels," Infection, vol. 70, no. 5, pp. 541-543, 2015.

[22] S. Y. Park, S.-O. Lee, S.-H. Choi et al., "Aspergillus galactomannan antigen assay in bronchoalveolar lavage fluid for diagnosis of invasive pulmonary aspergillosis," Infection, vol. 61, no. 6, pp. 492-498, 2010. 


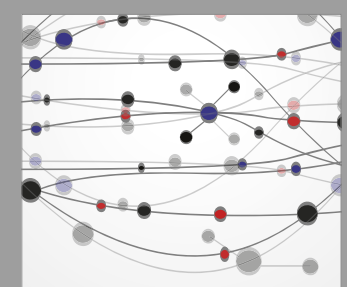

The Scientific World Journal
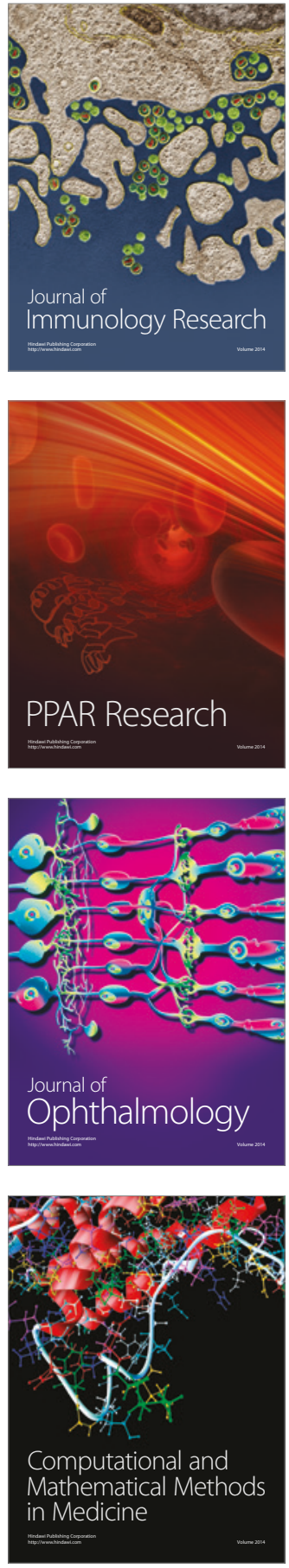

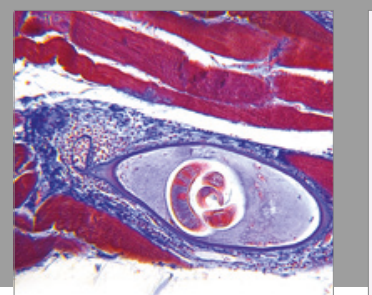

Gastroenterology Research and Practice
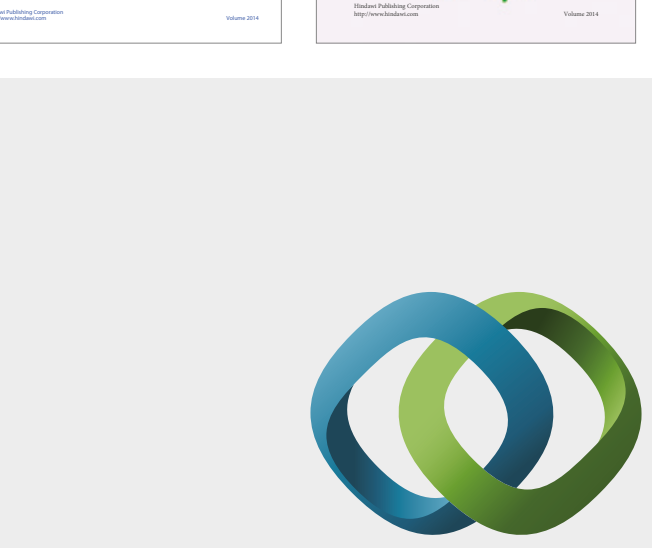

\section{Hindawi}

Submit your manuscripts at

https://www.hindawi.com
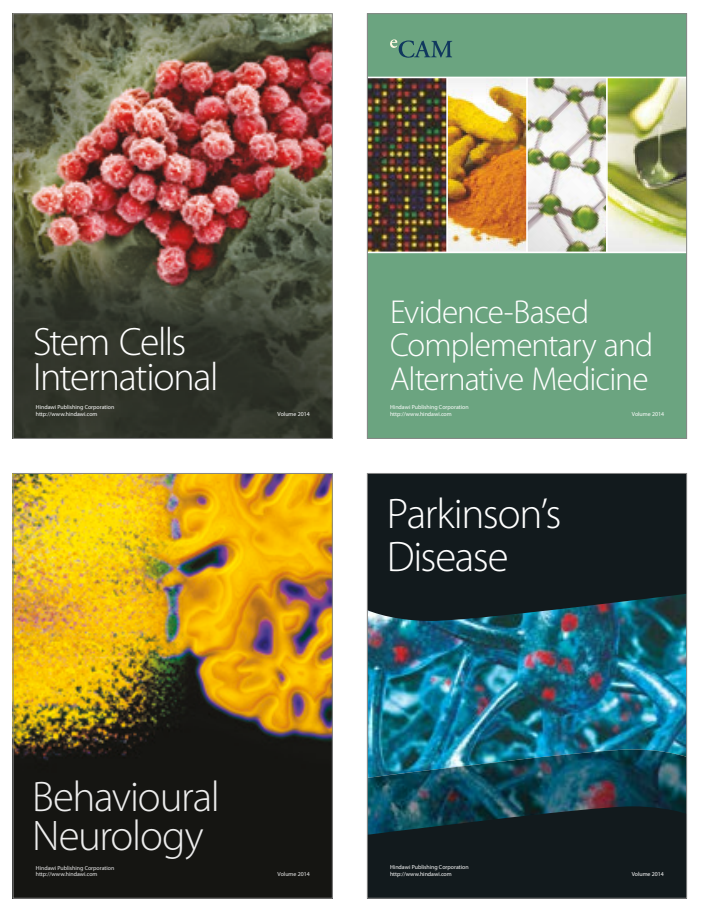
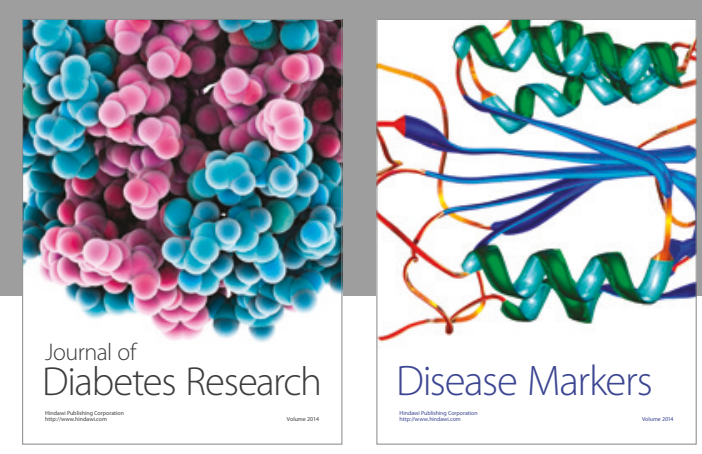

Disease Markers
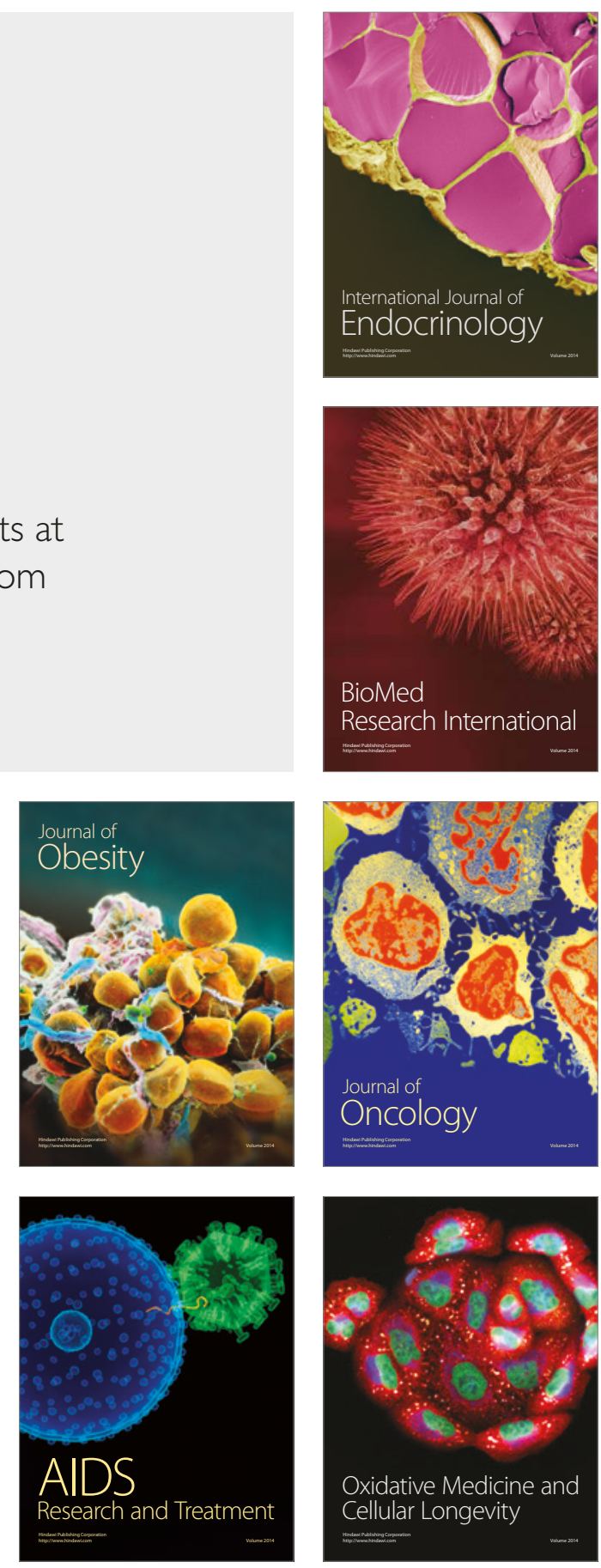